\title{
Dimensional mass customization of a flexible furniture system
}

XXIV International Conference

of the Iberoamerican Society

of Digital Graphics

Medellín | Colombia

\author{
Raquel Magalhães Leite \\ UFC/Unicamp | Brazil | raquelmleite@gmail.com \\ Gabriela Celani \\ Unicamp | Brazil | celani@unicamp.br
}

\begin{abstract}
This paper presents the development of a framework to investigate possible intersections between mass customization and flexibility in the furniture scale, based on the notion of complex modularity. The conceptual framework was implemented with parametric design as a prototypical furniture system, which allows dimensional customization and digital fabrication in reduced scale. The system was evaluated through workshops that simulated pre-configuration and reconfiguration layouts in a study room. Results showed that embedding complex modularity into furniture elements and defining measurement intervals contributed to the flexibility of the mass customized alternatives, providing the emergence of unexpected layout compositions and furniture uses.
\end{abstract}

Keywords: Mass customization; Parametric design; Furniture; Complex modularity.

\section{INTRODUCTION}

The concept of mass customization was firstly applied by Davis (1987) for fabricating unique products with mass production efficiency, being expanded into several steps along the manufacturing chain, from design to use (Duray, Ward, Milligan, \& Berry, 2000). In architecture, it has been strongly supported by parametric design and digital fabrication (Kolarevic 2005).

Modularity is recognized as a major mass customization strategy, as combining standard components provides low cost and quality control (Duray et al., 2000). Additionally, it fosters adaptability by accommodating change, like in the Future Adaptive Building replaceable cabinets (Brown, 2018). However, Salingaros and Tejada (2001) argue that modular designs tend to produce a narrow solution space. Therefore, modularity should not rely only on repeating similar components; instead, elements should be divided into smaller subparts and dimensions to achieve what Salingaros and Tejada (2001) call complex modularity, or modularity by subdivision.

Another mass customization possibility is dimensiona customization. This approach needs to comply with design regulations and ergonomics, and presents challenges when specifying parameters to avoid what Piller et al. (2005) called "mass confusion". Consequently, most mass customization systems apply a more traditional kind of modularity and limit users' choice (Khalili-Araghi \& Kolarevic, 2016). Alternatively, through parametric design, a constraint-based, dimensional customization system can generate "topologically similar but geometrically identical" results (Khalili-Araghi \& Kolarevic, 2016, p. 233). Accordingly, embedding complex modularity into design constraints could be a viable approach for mass customization, notably in the scale of spaces and objects.
On the other hand, it has been noted that an excessive amount of initial customization can lead to over-specified solutions, disregarding transformation demands over a building's life cycle (Schneider \& Till, 2007). The notion of flexibility or adaptability considers a certain amount of indeterminacy to accommodate changes in users' needs and context (Schmidt III \& Austin, 2016). In a building's inner layers, which comprise furnishings and spatial layout, different adaptability types may be employed, such as adjustability - change of task comprising object physical modification (Schmidt III \& Austin, 2016) and versatility change of use as a result of new interpretations, without physical intervention (Gu, Hashemian, \& Nee, 2004).

In this scenario, this paper aims to explore convergences between dimensional customization and complex modularity to achieve flexible solutions, as part of a master's research that investigated furniture dimensional customization in user-driven office layout compositions. It presents a framework for furniture dimensional customization based on the principles of mass customization, modularity and flexibility, applying parametric design and digital fabrication. Aligned to Simon's (1996) discussion on design science as a prescriptive discipline, we have developed a conceptual framework and implemented it with parametric design in Rhinoceros and Grasshopper.

The resulting furniture system was tested as an application study in a workshop. Reduced-scale models were produced with digital fabrication and manipulated by participants to create and reconfigure furniture and layout solutions. This experience allowed us to identify main flexibility features and their relationship to complex modularity, which had been embedded into the parametric furniture components. It also revealed limitations and refinements to be incorporated in subsequent iterative development cycles. 


\section{RESEARCH DEVELOPMENT CONCEPTUAL FRAMEWORK}

The starting point of framework development was to establish a reference context. In this research, we have focused on office furniture through the analysis of two cases: Herman Miller's workplace items by Alexander et al. (1987) and Opendesk (n.d.) furniture components.

As part of a study developed for the furniture company Herman Miller, Alexander et al. (1987) aimed to break modularity by allowing users to determine furniture dimensions during an office layout design process (Salingaros \& Tejada, 2001). Despite being conceived with a mass production background, their method already expressed mass customization concerns regarding processes and tools, "especially since the materials and finishes are unusual (at least by present industry standards). [...] It is possible that distribution and service of the furniture may need to be less centralized than most current systems" (Alexander et al., 1987, p. 140).

Opendesk, in turn, is an online platform based on open design principles that showcases several office furniture items to be bought or downloaded by users, who can digitally fabricate and assemble pieces on their own. Both Opendesk (n.d.) and Alexander et al. (1987) examples allow a defined amount of customization upon customer demand, but do not extend that flexibility to the furniture set as a system.

After analyzing furniture elements of both examples, we organized them according to how they can be used and identified the following prevailing activities: working (individually or in groups), having meetings, having a meal or a cup of coffee, storing, dividing space, communicating, sitting and making telephone calls. We observed that those demands could be fulfilled by three general furniture typologies: (1) horizontal elements, (2) vertical elements and (3) seating elements (Figure 1). They all can start from a simple box with a generic length (Lgth), depth (Dpth) and height $(H g h t)$ and become progressively distinct through dimensional constraints and addition or removal of planes. Besides, the typologies are versatile, which means that some objects can be used in several ways (e.g. a bookcase module can function as a side table or a stool).

After that, we consulted technical standards to define minimum and maximum dimensions to each part of different furniture items, as well as their relationships within the object's structure. Measurements were refined following Panero and Zelnik (1979) recommendations on anthropometrics. Additionally, furniture items can be generated and grouped in three different ways (Figure 2). (1) Single elements consist on individual furniture components obtained from the primary typology. (2) Matrix elements are based on the association of similar single items that together constitute an individual piece of furniture. Finally, (3) compound elements are the combination of two or more different single or matrix items to create furniture sets.
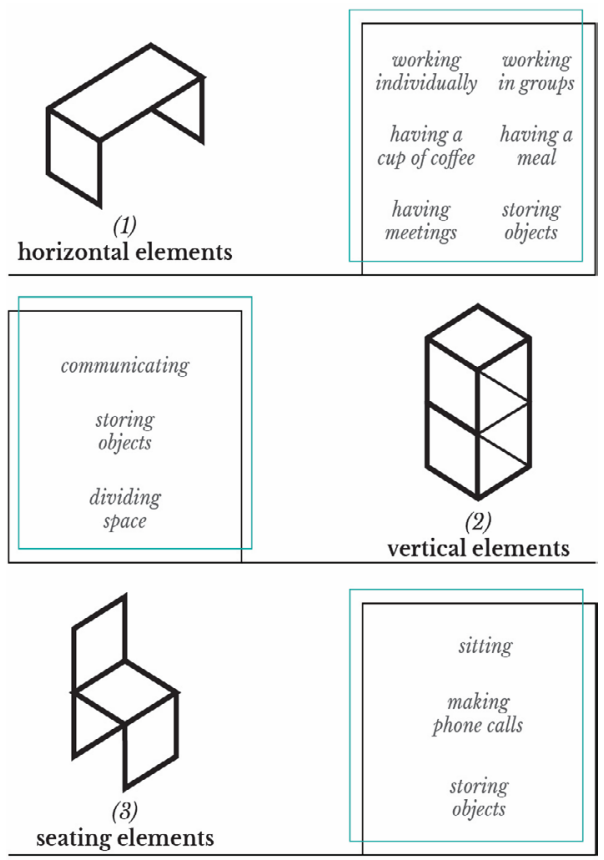

Figure 1: General furniture typologies and related activities. Source: authors.

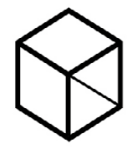

(1)

single element

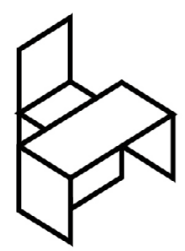

(3)

compound elements

Figure 2: Furniture groupings: (1) single element, (2) matrix element and (3) compound elements. Source: authors.

The last step consisted on applying complex modularity to the constraints that had been previously set. Usually, in a dimensional customization process, implemented with parametric design, numerical values are attributed continuously along an interval $\left[C v_{\min } ; C v_{\max }\right]$ of minimum and maximum measurements. Additionally, establishing interrelated values through associative geometry fosters adjustability among different furniture parts and avoids mass confusion that could result from an exceptionally high number of solutions. Thus, constraints were adjusted to a new interval $\left[M v_{\min } ; M v_{\max }\right]$, where $M v_{\min }$ and $M v_{\max }$ are multiples of a submodule Mod. It means that the number of 


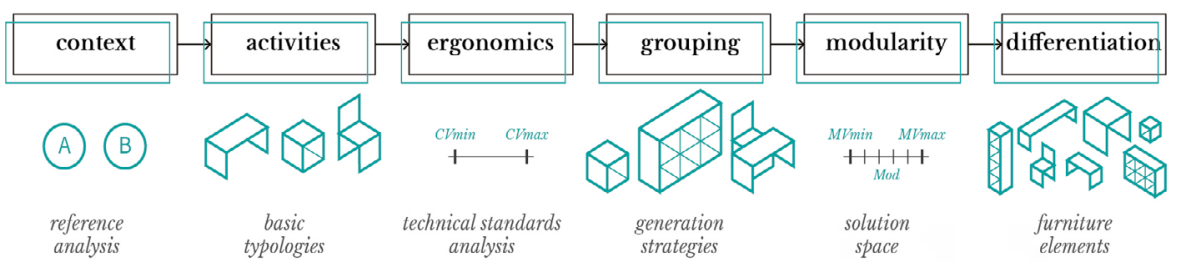

Figure 3: Conceptual framework overview. Source: authors.

solutions $s$ in each coordinate depends on the submodule value:

$$
s=\frac{\left(M v_{\max }-M v_{\min }\right)}{\operatorname{Mod}}+1
$$

Furthermore, in matrix elements, we had to determine values for the $(n \times p)$ rectangular array. For instance, in a bookshelf, after attributing Lgth, Dpth and Hght dimensions to a single box, one must set a number of rows $(n)$ and columns $(p)$. This is a simple example of modularity by subdivision: a complete bookshelf is composed by box modules, which, in turn, are created from multiples of a submodular dimension. The total number of solutions $S t$ is obtained by the product of the number of solutions in each coordinate and matrix lines and columns:

$$
S t=s_{L g t h} * S_{D p t h} * S_{H g h t} * s_{n} * s_{p}
$$

In compound elements, it was also necessary to take into account the necessary space to access and use furniture appropriately. This dimensional range is called clearance by Anderson et al. (2018) and, in this paper, it has been named aggregated area $(a A)$. Panero e Zelnik (1979) recommendations on anthropometry were used as a reference to define the aggregated area dimensional rules. For example, the authors suggest comfortable intervals to position a worktable and a chair according to a room's features, such as circulation routes or access to other furniture elements. In this case, the aggregated area $(a A)$ variable was also defined by applying dimensional intervals and, after that, adjusting those intervals to multiple numbers of a submodule. Figure 3 illustrates the overall framework process.

\section{PARAMETRIC FURNITURE SYSTEM}

The framework provided conceptual information for implementing a prototypical furniture system with parametric design tools and, subsequently, using digital fabrication machinery for producing prototypes.

The association between the three-dimensional modeling software Rhinoceros and the visual programming interface Grasshopper, which is based on the combination of variables according to an algorithmic thinking, enables the generation of geometries through parametric design processes. These were the tools chosen for the furniture system implementation.

Nine furniture items were derived from the three typologies and groupings initially defined: individual desk with a chair (iDsk), group table with chairs $(g T b /)$, table/stool (tStl), bench (Bnch), armchair (Armchr), sofa (Sofa), partition (Prttn), regular bookshelf ( $r B k s f)$ and dynamic bookshelf $(d B k s f)$. Object instantiation was done in four steps: (1) positioning, (2) general dimensioning, (3) specifying material and joints and (4) generating fabrication documents, as shown in Figure 4.
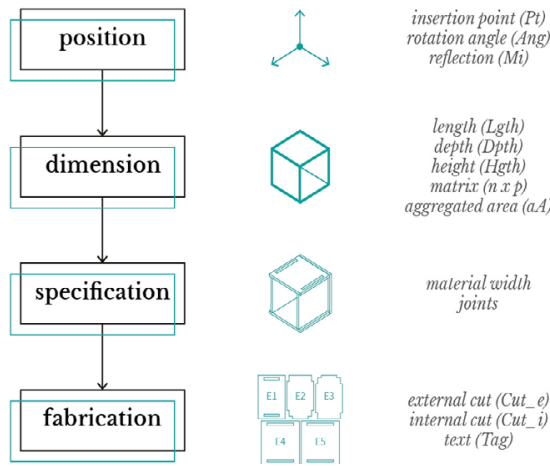

Figure 4: Parametric design workflow. Source: authors.

The parametric design process started with defining an object's position onto a three-dimensional space. An anchor point $P t(x, y, z)$ was set as a $X Y$ plane origin. This base plane was then connected to Grasshoppers' Domain Box component, which also requires numerical parameters as inputs to set dimensions of the box in each axis.

Thus, the second step consisted on attributing values in the $\mathrm{x}$-direction (Lgth), y-direction (Dpth) and z-direction (Hght). It was done with a Series component, which implements an arithmetic progression based on Start $\left(M v_{\min }\right)$, Step $(M o d)$ and Count (s) parameters. Series was used as a strategy to associate dimensional customization to complex modularity, establishing subdivision values (Mod) along an interval and, consequently, correlating solution spaces within the furniture family. In this implementation, Mod value was set to $150 \mathrm{~mm}$.

After that, the Domain Box was deconstructed into faces edges and vertices using the Deconstruct Brep component. Relevant faces were extracted as outputs to define furniture parts - for example, a table is generated by two side faces that act as supports; one upper face, the tabletop; and one side face for locking. If necessary, some faces can be scaled and moved to the appropriate position. The combination of all faces generates a preliminary geometry of a furniture piece.

For matrix elements, the resulting geometry was arranged into rows $(n)$ and columns $(p)$ to create another furniture piece, such as a bookshelf. In compound elements, on the other hand, the aggregated area $(a A)$ parameter was 

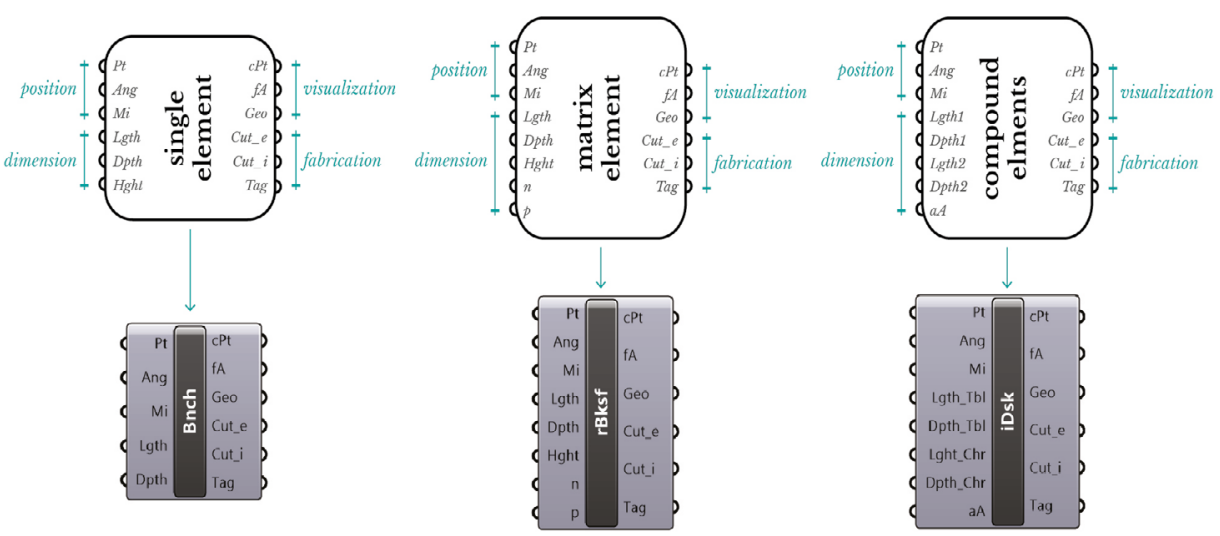

Figure 5: (1) Single, (2) matrix and (3) compound parametric elements with corresponding practical examples: (1) bench, (2) regular bookshelf and (3) individual desk. Source: authors.

included to define a distance ratio between objects. This distance was also set using the Series component.

The positioning and general dimensioning phases were concluded by adding rotating $(\mathrm{Ang})$ and reflecting $(\mathrm{Mi})$ features to the piece. The furniture geometry, the $X Y$ base plane and a Control Knob parameter ranging from 0 to 360 were attached to a Rotate component. For reflection, a Mirror component was used, controlled by a Boolean Toggle parameter.

In step three - specification - furniture parts were extruded by the chosen material width. During that phase, they were also altered according to manufacturing characteristics, such as joints' positions and sizes, with more precise value controllers. After that, Solid Difference components were applied to remove volumes between intersecting elements and output three-dimensional furniture parts.

Those solids were deconstructed and the respective contouring boundaries for each part were organized for digital fabrication. Pieces were re-dimensioned to the desired scale, tagged and organized within a twodimensional boundary using OpenNest, a Grasshopper plugin. Finally, the pieces were instantiated to the Rhinoceros window in separate layers - internal cut (Cut_i), external cut (Cut_e) and tag (Tag) - using the Grasshopper plug-in Human.

Each furniture element was synthetized into a Grasshopper Cluster, that receives positioning (Pt, Ang e Mi) and dimensioning (Lght, Dpth e Hght) parameters as inputs, and outputs a $3 \mathrm{D}$ visualization $(\mathrm{Geo})$, its central point projected on the ground $(c P t)$, floor area $(f A)$ and fabrication pieces in their respective layers (Cut_e, Cut i e $T a g$ ). Matrix items also include the number of rows $(n)$ columns $(p)$ as inputs, and compound elements, the aggregated area $(\mathrm{aA})$. Figure 5 illustrates the three kinds of Clusters with corresponding practical examples.

Finally, 1:20 prototypes of different dimensionally customized variations of each piece of furniture were lasercut and assembled as a proof of concept (Figure 6). Dimensional controllers made it easy to set general and specific measurements in order to generate a variety of solutions. The joint details employed were very simple, in order to focus on the overall logic that pervaded all the elements within the furniture set. In full scale applications, more sophisticated joints may be used.

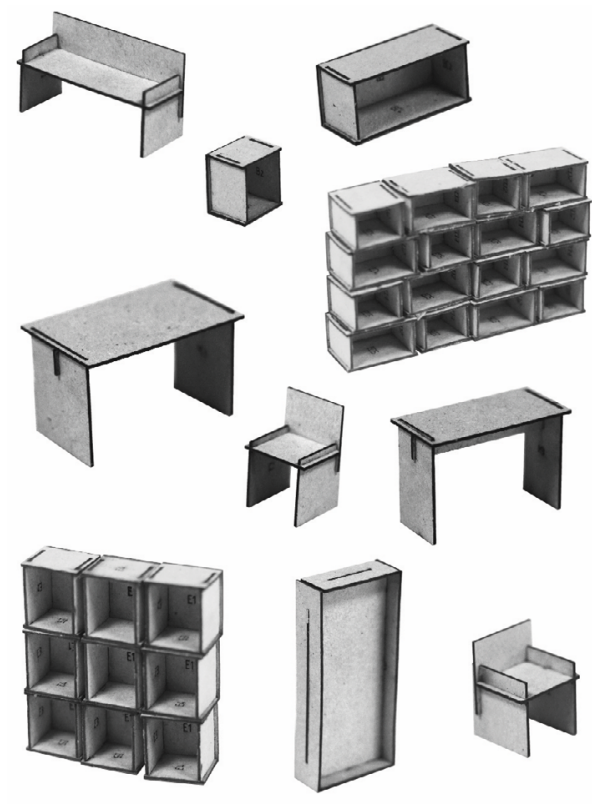

Figure 6: 1:20 furniture prototypes. Source: authors.

\section{APPLICATION STUDY}

The final step of this development cycle was to test the prototypical furniture system in a practical context. During the master's research, an application study was conducted through a series of workshops with different groups. One of them is presented in this paper, focusing on flexibility features that were achieved with the system. Because it involved human interaction, the research project was 

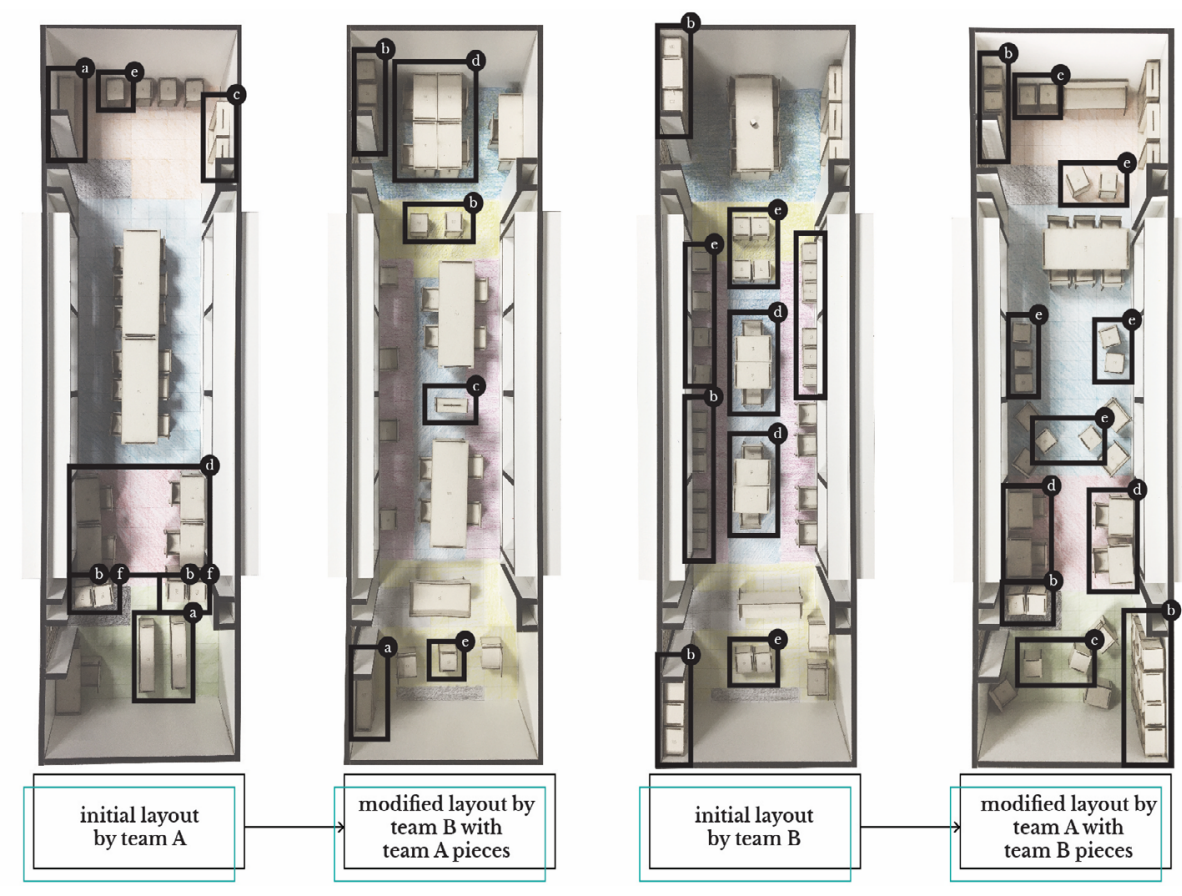

Figure 7: Pre-configuration and reconfiguration layouts to a study room, developed by Team A and Team B, as a system's application study. Source: authors.

evaluated and approved by Unicamp's Research Ethics Committee (CEP-CHS, CAAE: 11693819.1.0000.8142).

This particular workshop was directed to trained architects, who were asked to create a layout to a study room using the customizable furniture set. Four participants, divided in two teams (Team A and Team B), used the parametric tools within Rhinoceros and Grasshopper environments to digitally design a spatial layout that should accommodate the following activities: reading and writing, studying in groups, making a pause and using digital media. By the end of the exercise, pieces of the designed furniture elements were laser-cut in 1:20 scale, assembled and positioned into a physical model by the participants.

After that, each team was challenged to use the furniture elements created by the other team to compose new layouts, still seeking to satisfy their original demands. During that phase, only the physical model was used. The experience enabled to simulate the system's adaptability to changes in circumstances - in that case, new users with different spatial requirements.

The moments of pre-configuration and reconfiguration were compared according to the above-mentioned adaptability types: adjustability and versatility. Figure 7 summarizes initial and modified layouts created by each team, with highlights on the main flexible solutions applied by participants.

The adjustability feature was verified by physical changes in furniture pieces from pre-configuration to reconfiguration phases. Those changes could be a result of disassembly and reassembly of parts - or complete modules in matrix elements - that generated new furniture types. Adjustability was applied, for example, by stacking benches to generate a bookcase (a) and by disassembling a bookcase to create a new one with a different number of rows and columns, or several table/stools (b). The use of modularity by subdivision made it easier to rearrange modules in new compositions, that were consistent with the spatial organization of the room.

The other flexibility type, versatility, was identified in layout compositions from the perception of affordances (Gibson, 1979) by participants, that is, using a single object in different ways without any physical change in its parts. Versatility examples were found in the panel being applied as a blackboard and a partition (c), individual desks being used separately or grouped (d), the table/stool in both configurations (e), and bookshelves serving as room partitions (f). In general, single elements provided more versatility, was well as desks with smaller widths.

\section{DISCUSSION}

This paper presented a framework based on the application of complex modularity for defining measurements of a flexible furniture set, within an interval based on ergonomic standards, through a parametric design workflow. This strategy enabled to embed appropriate constraints in the dimensional customization process and to define a coherent design space within the whole furniture family. Besides, the system's logic made furniture pieces easy to customize and fabricate under the mass customization paradigm. 
In the application study developed through a workshop with architects, flexibility was verified by the emergence of two features: adjustability and versatility. Reconfigured layouts simulated the need for change resulting from new demands and revealed that modularity by subdivision, which had been embedded into furniture pieces during the primary design phase, promoted adjustable and versatile solutions. The study suggests that life-long adaptability could be achieved by the perception of affordances by participants and by the retrofit of some components when changing the space layout, which would be possible due to the assembly/disassembly strategies and to the dimensional articulation between submodules.

The experience also raised questions regarding authorship and responsibility along the design workflow. When proposing a dynamic system instead of a finished product, designers have even more responsibility in specifying design constraints, as they need to comply with a series of possible solutions. At the same time, authorship is shared between designer, user and software, making it important not to over-determine parameters at a point that limits users' intervention. In that sense, the act of customizing can also be seen as part of a creative and learning environment.

Future work could encompass the system's detailing, especially in terms of materials, joints and other manufacturing specifications, as well as the development of a user-friendly interface. Additionally, furniture parametric components could be improved with topological customization, by embedding relational characteristics linked to dimensional ones.

The proposed framework brings a possibility for layout customization that goes beyond furniture arrangement or modularization. Even with a simplified furniture set and constraints definition based on ergonomic requirements, the reconfiguration activity showed a satisfactory degree of customization, where participants were able to overcome some initial limitations and achieve new shapes and activities. We hope that the research findings will contribute to the development of new parametrically adaptable furniture solutions.

\section{ACKNOWLEDGMENTS}

This study was financed in part by the Coordenação de Aperfeiçoamento de Pessoal de Nível Superior - Brasil (CAPES) - Finance Code 001.

We would also like to thank the participants of the workshops for their interest and involvement, the Laboratory of Automation and Prototyping for Architecture and Construction (LAPAC/Unicamp) for providing the infrastructure for the workshops, the Unicamp Student
Housing for the partnership, and Caio Castriotto for his assistance in implementing the Grasshopper definitions.

\section{REFERENCES}

Alexander, C., Anninou, A., Black, G., \& Rheinfrank, J. (1987). Toward a personal workplace. Architectural Record, 175(11), 130-141.

Anderson, C., Bailey, C., Heumann, A., \& Davis, D. (2018). Augmented space planning: Using procedural generation to automate desk layouts. International Journal of Architectural Computing, 16(2), 164-177. https://doi.org/10.1177/1478077118778586

Brown, J. L. (2018). Future adaptive building: Mass-customized housing for an aging population. In B. Kolarevic \& J. P. Duarte (Eds.), Mass Customization and Design Democratization (1st ed., pp. 185-196). New York: Routledge.

Davis, S. M. (1987). Future Perfect (1st ed.). Reading: Addison Wesley.

Duray, R., Ward, P. T., Milligan, G. W., \& Berry, W. L. (2000). Approaches to mass customization: Configurations and empirical validation. Journal of Operations Management, 18(6), 605-625. https://doi.org/10.1016/S02726963(00)00043-7

Gibson, J. J. (1979). The ecological approach to visual perception. Boston: Houghton Mifflin.

Gu, P., Hashemian, M., \& Nee, A. Y. C. (2004). Adaptable Design. CIRP Annals, 53(2), 539-557. https://doi.org/10.1016/S00078506(07)60028-6

Khalili-Araghi, S., \& Kolarevic, B. (2016). Development of a framework for dimensional customization system: A novel method for customer participation. Journal of Building Engineering, 5, 231-238. https://doi.org/10.1016/j.jobe.2016.01.001

Kolarevic, B. (Ed.). (2005). Architecture in the Digital Age: Design and Manufacturing (1st ed.). New York: Taylor \& Francis.

Opendesk. (n.d.). Opendesk - Furniture designed for inspiring workplaces. Retrieved May 25, 2019, from https://www.opendesk.cc/

Panero, J., \& Zelnik, M. (1979). Human Dimension \& Interior Space: A Source Book of Design Reference Standards (Revised ed.). New York: Watson-Guptill.

Piller, F., Schubert, P., Koch, M., \& Möslein, K. (2005). Overcoming Mass Confusion: Collaborative Customer CoDesign in Online Communities. Journal of Computer-Mediated Communication, 10(4). https://doi.org/10.1111/j.10836101.2005.tb00271.x

Salingaros, N. A., \& Tejada, D. M. (2001). Modularity and the Number of Design Choices. Nexus Network Journal, 3(1), 99109. https://doi.org/10.1007/s00004-000-0008-z

Schmidt III, R., \& Austin, S. (2016). Adaptable Architecture: Theory and practice (1st ed.). New York: Routledge.

Schneider, T., \& Till, J. (2007). Flexible Housing (1st ed.). Oxford: Architectural Press.

Simon, H. A. (1996). The Sciences of the Artificial (3rd ed.). Cambridge: MIT Press. 